\title{
Multichroic bandpass seashell antenna with cold-electron bolometers for CMB measurements
}

Leonid S. Kuzmin

Alexander V. Chiginev 


\title{
Multichroic Bandpass Seashell Antenna with Cold-Electron Bolometers for CMB Measurements
}

\author{
Leonid S. Kuzmin*a,b and Alexander V. Chiginev ${ }^{\mathrm{b}, \mathrm{c}}$ \\ ${ }^{a}$ Chalmers University of Technology, 41296 Gothenburg, Sweden; ${ }^{b}$ Dept. Nizhny Novgorod State \\ Technical University, 603155 Nizhny Novgorod, Russia; ' Institute for Physics of Microstructures, \\ Russian Academy of Science (RAS), 603087 Nizhny Novgorod, Russia.
}

\begin{abstract}
A novel type of the multichroic "seashell" resonant antenna is developed for CMB measurements. The polarized slot antennas are arranged in the compact form of a seashell with individual slots for each frequency and each polarization. Such an arrangement gives unique opportunity for independent adjusting individual parameters of slots with microstrip lines (MSL) and bolometers. For each frequency band the seashell antenna contains two pairs of orthogonal slots for each polarization connected by microstrip lines (MSL) with a bolometer in the middle for in-phase operation. To fit slots in $\lambda / 2$ area for the best beam shape, lumped capacitances in the form of H-slot were introduced. Ellipticity of a beam was improved to the level of better than $1 \%$.

The seashell antenna gives a unique opportunity to select needed bandwidth by resonant properties of slots themselves. Slots are phased by MSLs connecting two opposite slots with a resistive Cold-Electron Bolometer (CEB) placed just in middle of two MSLs. MSLs and CEBs are placed just in the area of the seashell antenna. The resonant seashell antenna with CEBs avoids long MSLs bringing signal outside the antenna to large external filters as in the case of sinuous antenna. This innovation avoids losses in long MSLs and increases frequency range.
\end{abstract}

Keywords: resonant seashell slot antenna, cold-electron bolometer (CEB), microstrip line (MSL), lumped capacitance, capacitance of the SIN tunnel junction

\section{INTRODUCTION}

The success of three generations of space missions and balloon and ground based telescopes dedicated to the study of the Cosmic Microwave Background (CMB) has delivered invaluable insight into the origins of the Universe and the physics of its earliest moments. It has founded a global cosmological model described by the expansion rate, various forms of matter and energy, spatial curvature, and primordial perturbations generated during a period of inflationary expansion. The model's free parameters are now measured to percent-level precision. This remarkable success sharpens attention on a number of fundamental questions. What are the natures of dark matter and dark energy, accounting for $96 \%$ of the energy density of the Universe? Whether a phase of cosmic inflation actually seeded the initial perturbations awaits full demonstration, and the mechanisms that initiated inflation are not well understood or constrained. Future observations of the CMB will validate or challenge this standard cosmological model, answer these open questions and provide further constraints on the fundamental properties of matter and interactions at energy scales up to twelve orders of magnitude higher than those attainable at the LHC. To exploit this exceptional potential, instruments must be designed with unprecedented performance.

Recently, the detection of gravitational waves was announced as a result of the American experiment BICEP2 (Background Imaging of Cosmic Extragalactic Polarization) [1]. Gravitational waves formed in the early evolution of the Universe, had to leave their marks in the vortex B-mode polarization of the CMB in accordance with the existing theoretical concepts. B-mode was observed by a phased array of slot antennas. However, these results are in contradiction with dust measurements of Planck [2] and should be checked at different frequencies.

*kuzmin@chalmers.se

Millimeter, Submillimeter, and Far-Infrared Detectors and Instrumentation for Astronomy VIII,

edited by Wayne S. Holland, Jonas Zmuidzinas, Proc. of SPIE Vol. 9914, 99141U

(C) 2016 SPIE · CCC code: 0277-786X/16/\$18 · doi: 10.1117/12.2232895

Proc. of SPIE Vol. $991499141 \mathrm{U}-1$ 
The measurement of the CMB polarization is included in the ambitious COSMIC VISION 2015-2025 program of the European Space Agency (ESA). The CORE+ and SPICA space missions are under consideration by ESA. The important goal for the ESA is to reduce size of the focal plane by placement of the multi-frequency array of detectors for simultaneous data acquisition [3]. Such an approach would solve the problem of the aberration and uniformity of the beam pattern across the total focal plane.

As a promising candidate of the multichroic systems, a novel "seashell" slot antenna has been proposed for this goal [4] (Fig. 1). The antenna is called seashell due to the similarity of round loops of slots for different frequencies with a seashell. The seashell slot antenna is the multichroic system which has several key advantages compared to other concepts: sinuous [5], and cross-slot [6,7]. The main advantage of the seashell antenna in comparison with sinuous and cross-slot antennas is that it provides the opportunity to tune independently separate slots for each frequency. It is also quite compact and can be easily extended to a larger number of frequency channels by adding proper slots. Moreover, this extension will not increase the size of the system because all new slots will be positioned inside the larger slots.

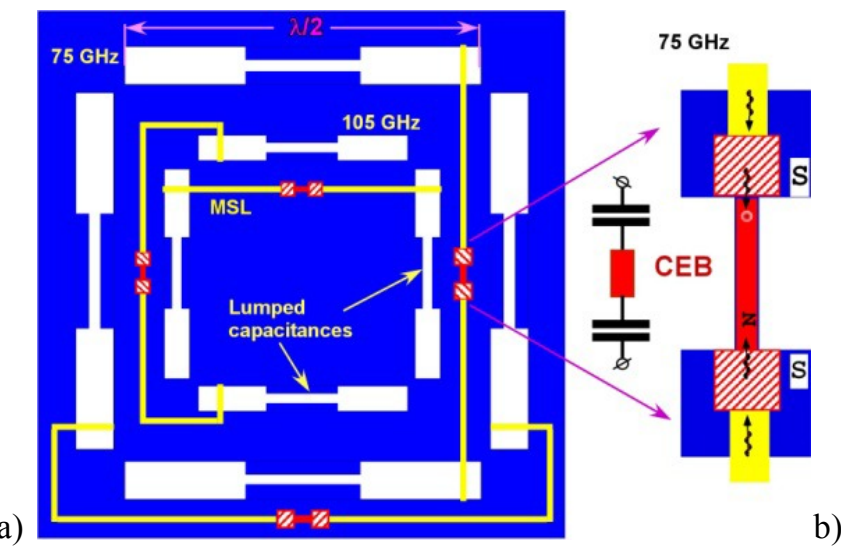

Figure 1. The $\lambda / 2$ seashell antenna for 75 and $105 \mathrm{GHz}$ with lumped capacitances, MSLs and CEBs; (b) a sketch of the CEB with an equivalent scheme.

The first suggestion for frequency selection was to use the recently invented resonant cold-electron bolometer (RCEB) [6] in combination with seashell antenna. An internal nanofilter of the RCEB is organized by a kinetic inductance of the $\mathrm{NbN}$ superconducting nanostrip and a capacitance of the nanoscale SIN (Superconductor-Insulator-Normal) tunnel junction. This internal resonance acts as a bandpass filter with a bandwidth of 3-50\% needed for radioastronomy applications.

However, in process of analysis we discovered that frequency selection can be organized by resonant properties of slots themselves. Important step in this realization was introducing lumped capacitances creating more narrowband resonances of the slots. Slots are phased in this case by MSLs with a resistive Cold-Electron Bolometer (CEB) [8-10] in the middle. In the present paper we report on analysis of the real model of a seashell antenna using MSLs losses and CEBs with final capacitances, designed for two frequencies -75 and $105 \mathrm{GHz}$.

\subsection{Seashell antenna including losses in MSLs and capacitance of CEBs}

A sketch of the seashell antenna for two frequencies used for simulation is shown in Fig. 2a. It consists of 4 slot antennas: two antennas for each frequency of 75 and $105 \mathrm{GHz}$ and for each polarization. Discrete ports are placed in the central part between two MSL to phased operation of opposite slots. Feeding of signal is shifted from the center of slots to the end to reduce the matching impedance with MSL and CEB to the level of 20-40 Ohm. Parameters of slots and ports position were determined for each frequency separately. To focus the beam, $9 \mathrm{~mm}$ silicon lens with antireflection coating of quartz with thickness of $430 \mu \mathrm{m}$ is used (Fig. 2b). We model CEBs as CR circuits with frequencies tuned to desirable channels. 

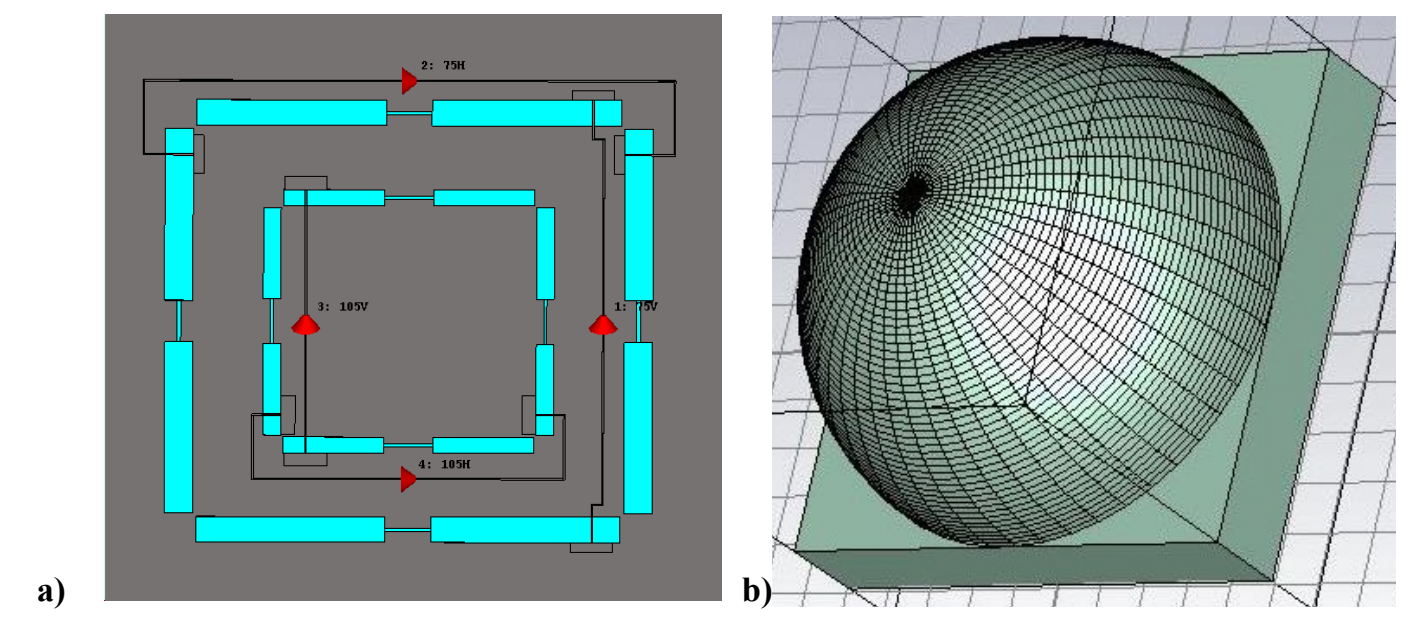

Figure 2. A sketch of the seashell antenna used for simulation with ports in CEB places for excitation; (b) A general view of the system with a lens and Si substrate;

Here we show the results of calculations of radiation diagrams and frequency properties of the seashell antenna. For numerical modeling we use CST Microwave Studio. To model a CEB we use serial connection of a port and discrete capacitance. We place this combination between two MSLs feeding opposite slots. In calculations we take into account losses in metal in ground plane and MSLs. We assume the metallic parts of the antenna to be made of gold and use the value of gold conductivity $\sigma=4.5 \cdot 10^{8} \mathrm{Ohm}^{-1} \mathrm{~m}^{-1}$.

\section{A. Radiation characteristics}

Here we present the results of modeling the radiation diagrams of the seashell antenna. We restrict ourselves only to consideration of slots for horizontal polarization, assuming that for vertical polarization the results are similar due to symmetry of the antenna.

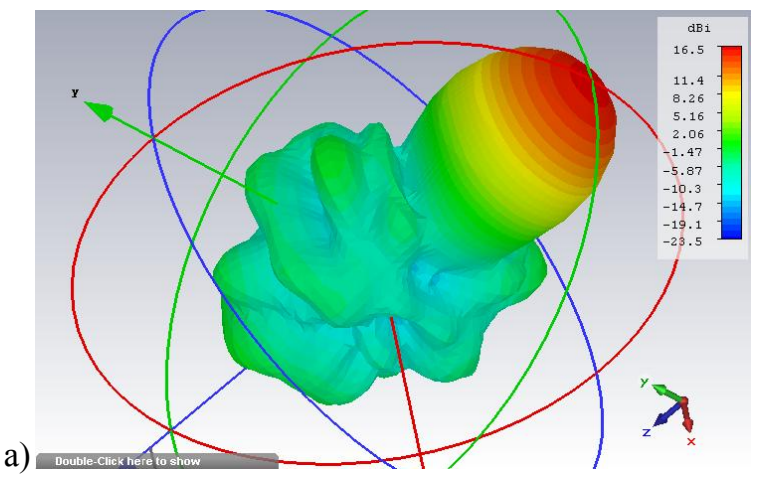



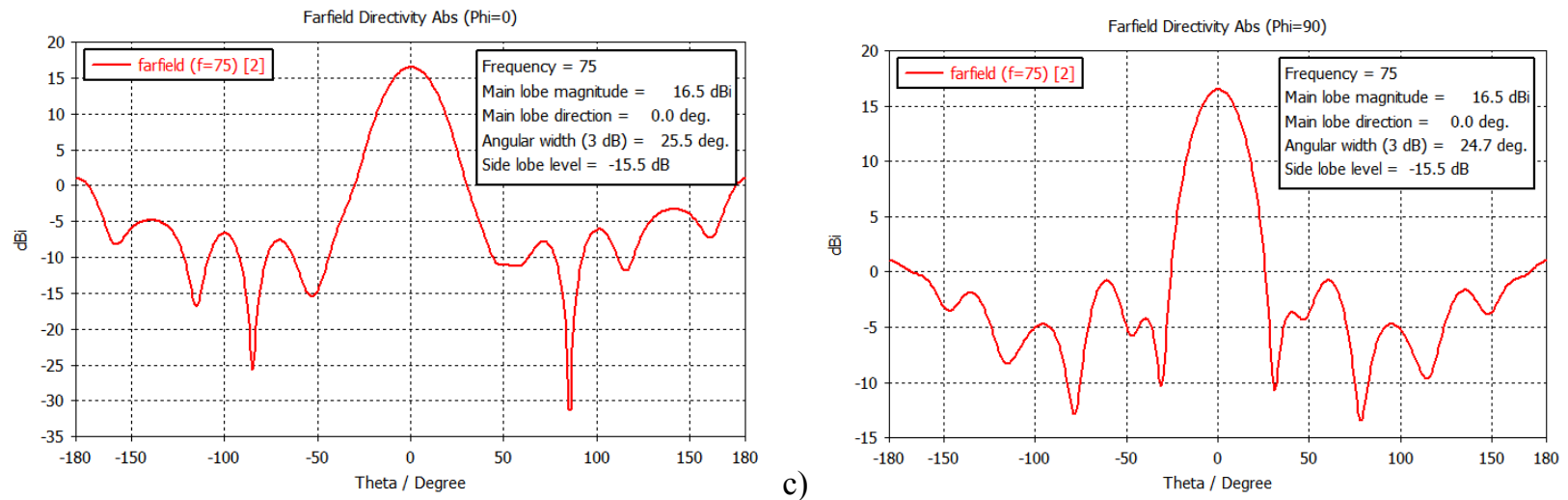

Figure 3. Radiation diagrams of the seashell antenna at 75 GHz: (a) a 3D view; (b) cross-section at $\phi=0$ deg.; (c) crosssection at $\phi=90 \mathrm{deg}$.
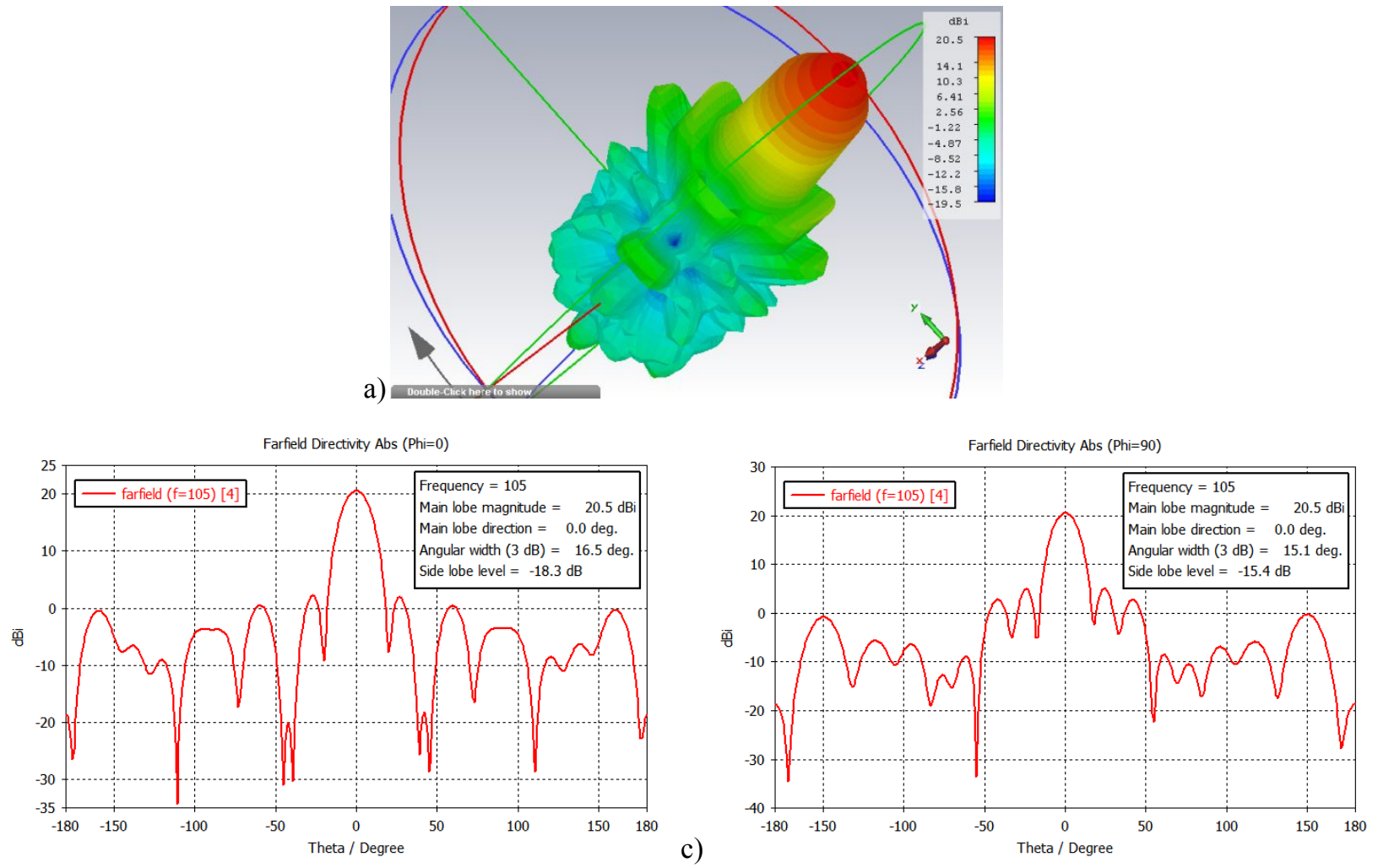

Figure. 4. Radiation diagrams of the seashell antenna at $105 \mathrm{GHz}$ : (a) a 3D view; (b) cross-section at $\phi=0$ deg.; (c) crosssection at $\phi=90 \mathrm{deg}$.

Figs. 3,4 show radiation diagrams of the seashell antenna for 75 and $105 \mathrm{GHz}$ channels, respectively. The angular width of the beam is $25.5 \mathrm{deg}$. and $16.5 \mathrm{deg}$. at 75 and $105 \mathrm{GHz}$. The beam ellipticity is $1.6 \%$ and $4.4 \%$ at 75 and $105 \mathrm{GHz}$. These values are quite good for COrE application. The calculation of cross-polarization suppression also give good values: $-22 \mathrm{~dB}$ at $75 \mathrm{GHz}$ and $-35 \mathrm{~dB}$ at $105 \mathrm{GHz}$. Side lobe levels are $-15.5 \mathrm{~dB}$ at $75 \mathrm{GHz}$ and $-15.4 \mathrm{~dB}$ at $105 \mathrm{GHz}$. To conclude, seashell antenna with lens provides rather good beam characteristics.

\section{B. Frequency characteristics}

For calculation of frequency characteristics of the seashell antenna we assume the value of the capacitance of the tunnel junction to be equal to $200 \mathrm{fF}$. The impedance of port is set to $20 \mathrm{Ohm}$. These values are realistic for CEB. We see that 
H-slot provide resonant frequency dependencies of S-parameters of the antenna (Fig. 5a). However, due to Ohmic losses in metal, the non-resonant absorption occurs. It is especially noticeable on S-parameter curve for $105 \mathrm{GHz}$ slots. Owing to this, it is difficult to estimate the FWHM of the resonances by usual way.

a)
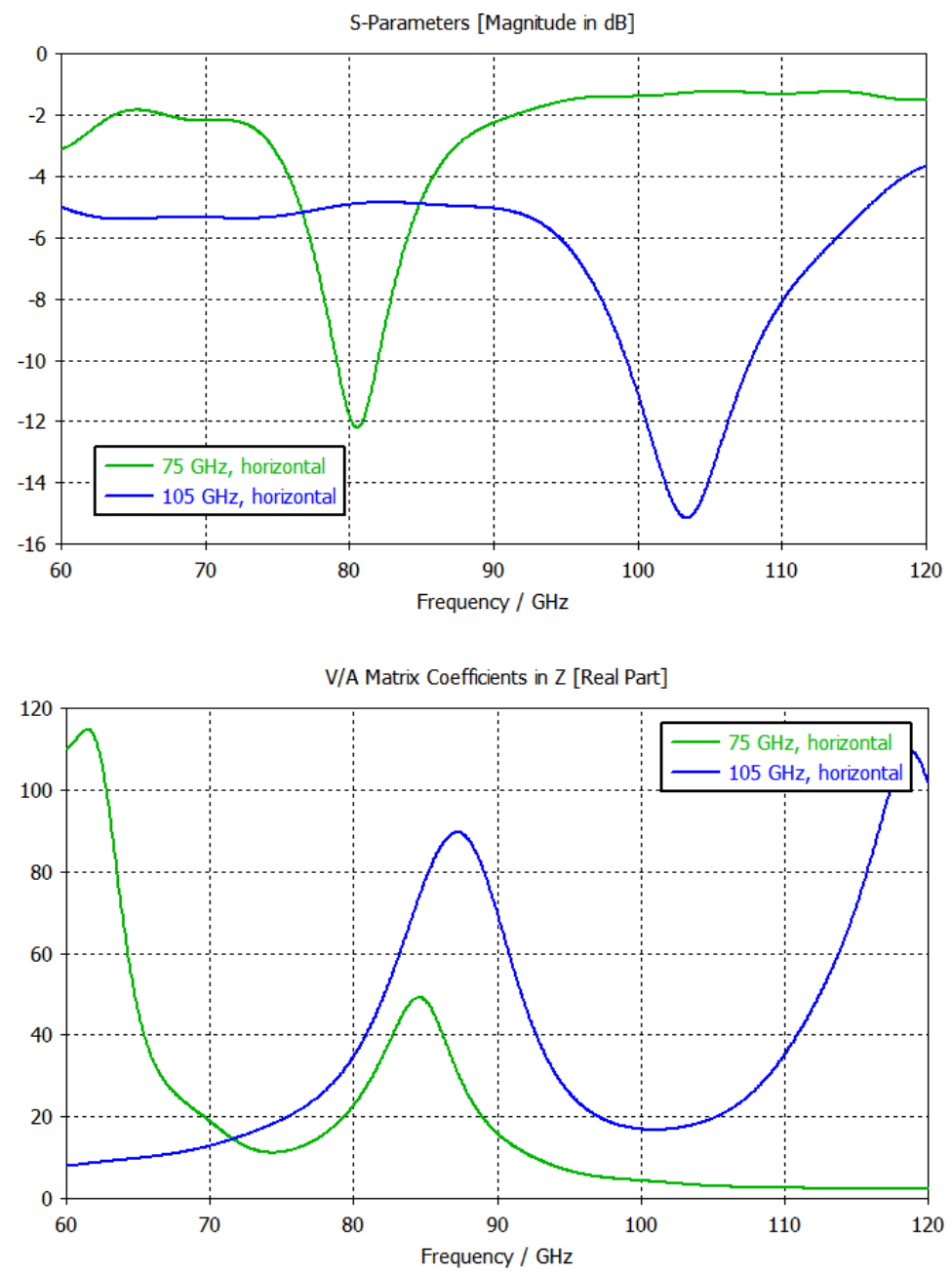

b)

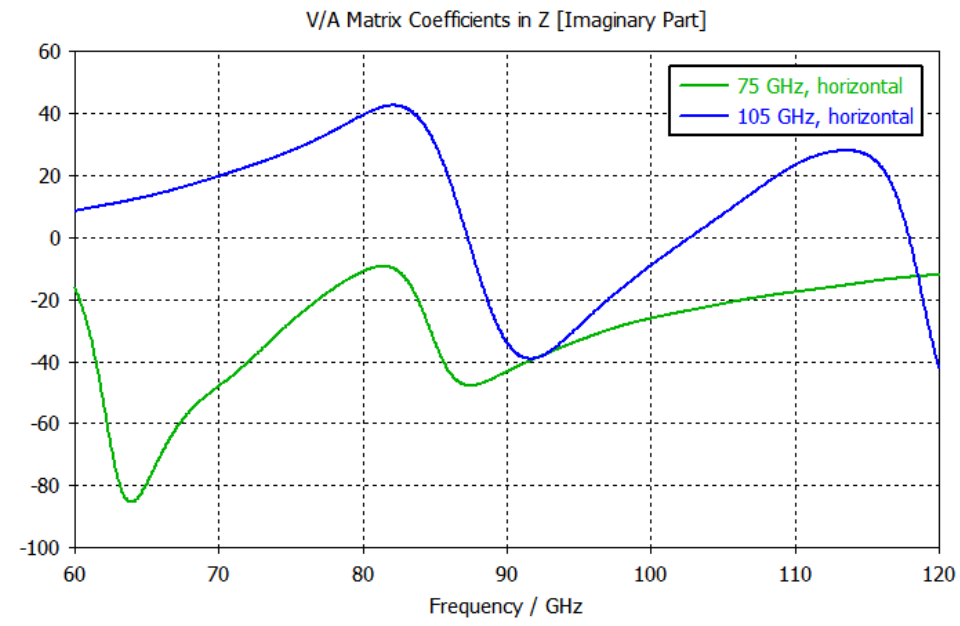

Figure. 5. (a) S-parameter of $75 \mathrm{GHz}$ slots and $105 \mathrm{GHz}$ slots; (b) ReZ and (c) ImZ of $75 \mathrm{GHz}$ slots and $105 \mathrm{GHz}$ slots. 
From Fig. $5 \mathrm{c}$ we see that the resonance in $105 \mathrm{GHz}$ channel is a serial resonance with $\mathrm{ImZ}=0$. This serial resonance is located between two parallel resonances, which are determined by geometrical sizes of the slots and MSL lengths.

\section{A. Losses in MSLs}

The Fig. 6 illustrates the influence of losses in metal on resonance structure. We see that at lower $\sigma$ the frequency dependencies of ReZ and ImZ become less pronounced (Fig. 6b,c). At the same time, the position of the serial resonance almost does not change (Fig. 6c). On the other hand, we see that ReZ increases near serial resonance with $\sigma$ decrease, and gets closer to the port impedance which is set to be $25 \mathrm{Ohm}$ (Fig. 6b). This is why S-parameter has deeper resonance at lower $\sigma$, and by the same reason another smooth resonance at lower frequencies appears.

a)
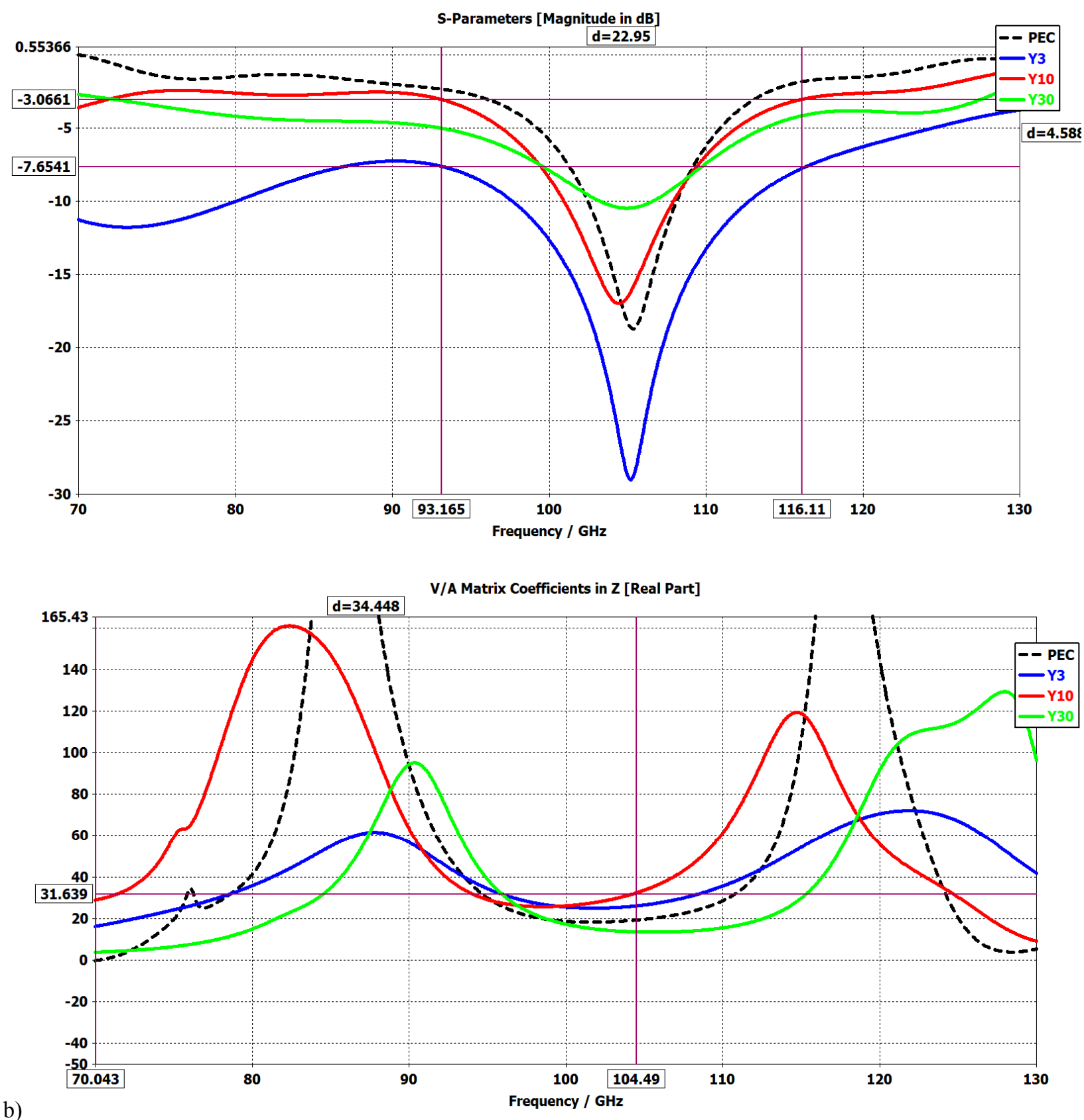


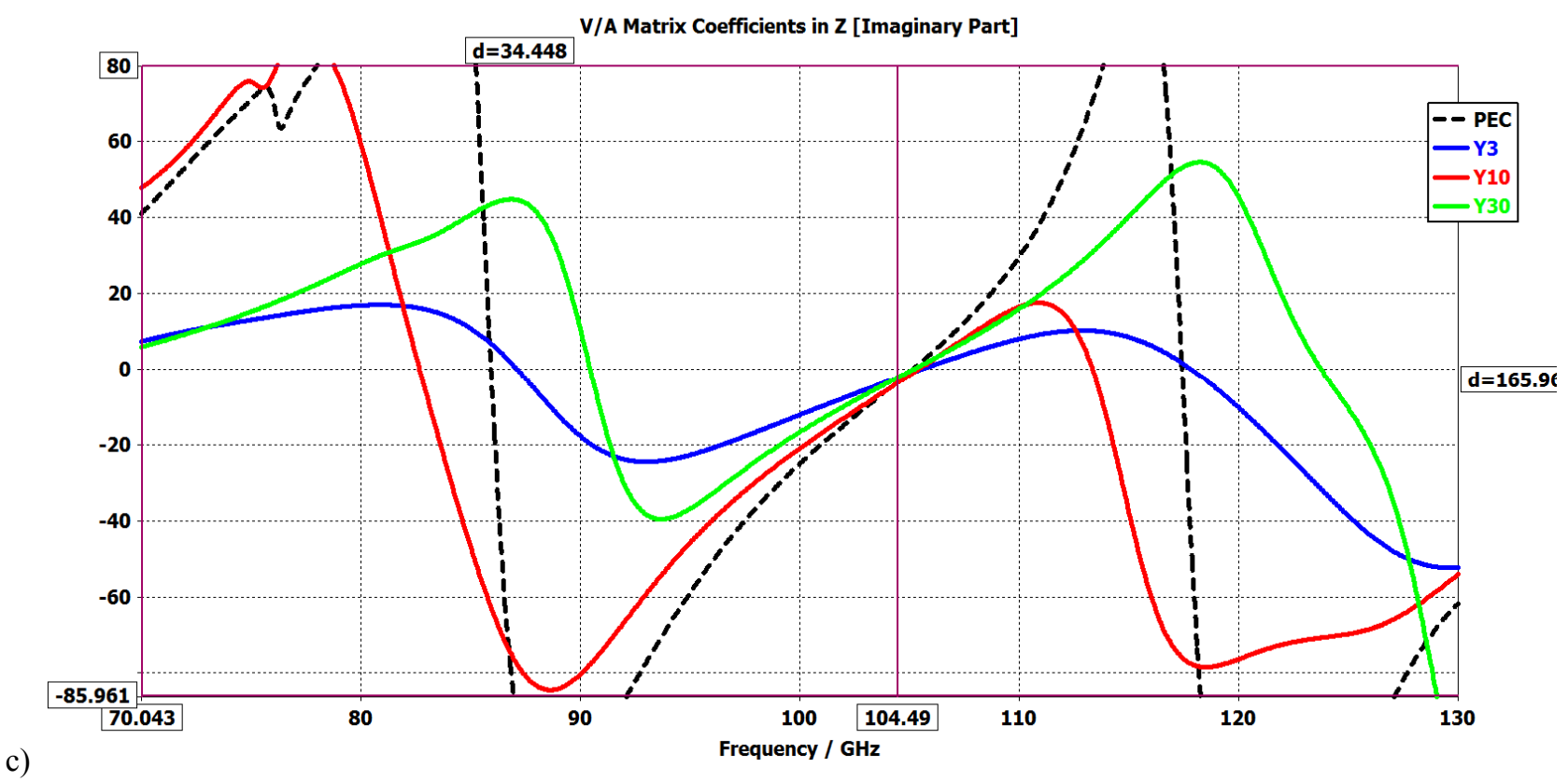

Figure 6. The influence of losses in metal on resonance structure. (a) S-parameters; (b) ReZ; (c) ImZ, plotted at various $\sigma$. Y3: $\sigma=1.5 \cdot 10^{8} \mathrm{Ohm}^{-1} \mathrm{~m}^{-1}, \mathrm{Y} 10: \sigma=4.5 \cdot 10^{8} \mathrm{Ohm}^{-1} \mathrm{~m}^{-1}$, Y30: $\sigma=1.5 \cdot 10^{9} \mathrm{Ohm}^{-1} \mathrm{~m}^{-1}$,

\section{$B$. Influence of the CEB capacitance}

The Fig. 7 illustrates the effect of capacitance in CEB to frequency characteristics of the antenna. We see that with capacitance decrease, the resonance shifts to higher frequencies with its width decrease. At the same time, the decrease in CEB capacitance leads to appearance of another resonance at lower frequencies (Fig. a). The latter can be explained by the impedance of the capacitor which has the form $Z_{C}=1 / i \omega C$ which is added to the antenna impedance and inevitably lowers the ImZ curve at lower frequencies (Fig. 7c). It means that another serial resonance appears to the left of the main parallel resonance. The position of this serial resonance depends on $\mathrm{C}$ and shifts to the right with $\mathrm{C}$ decrease.

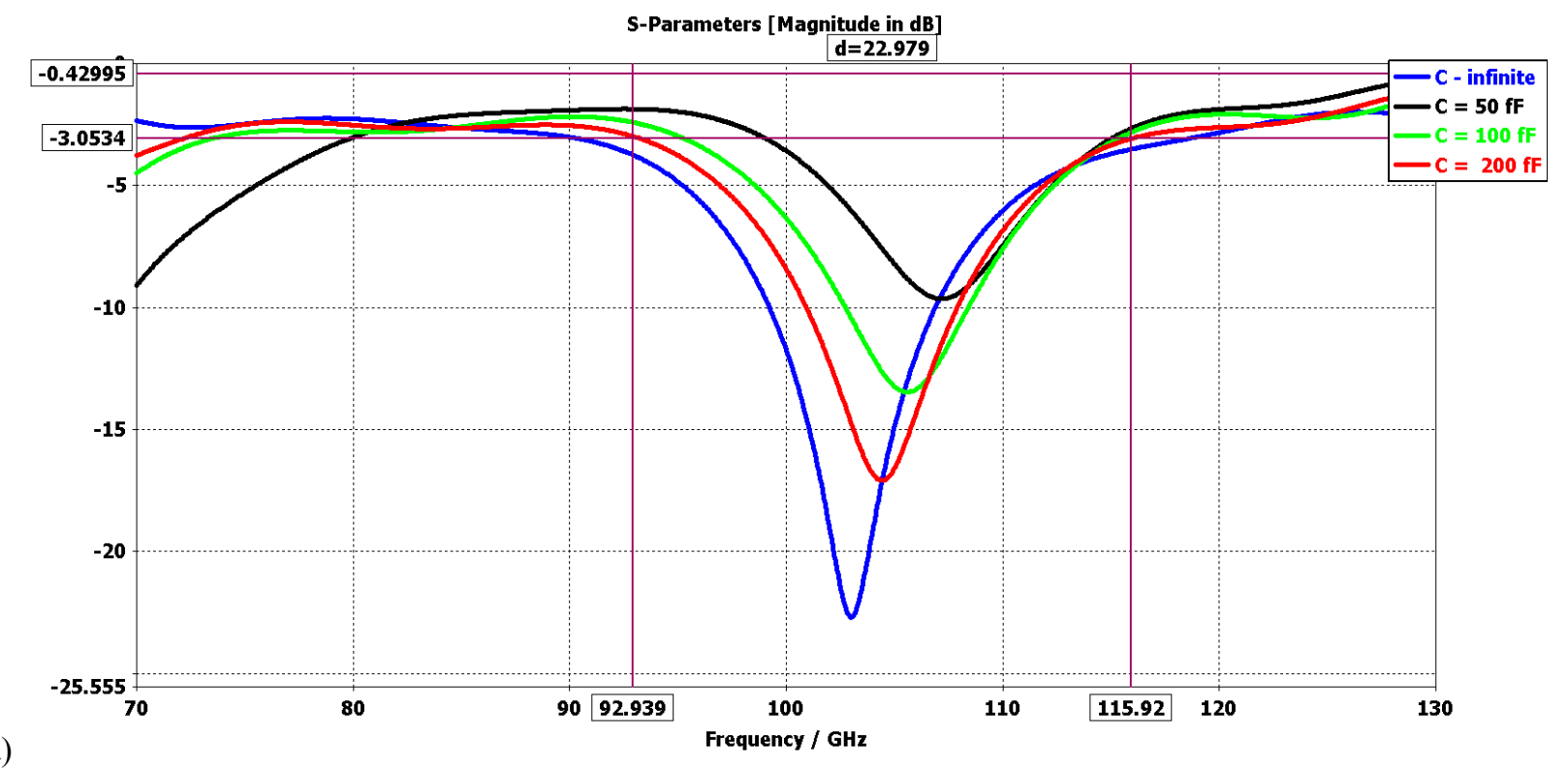




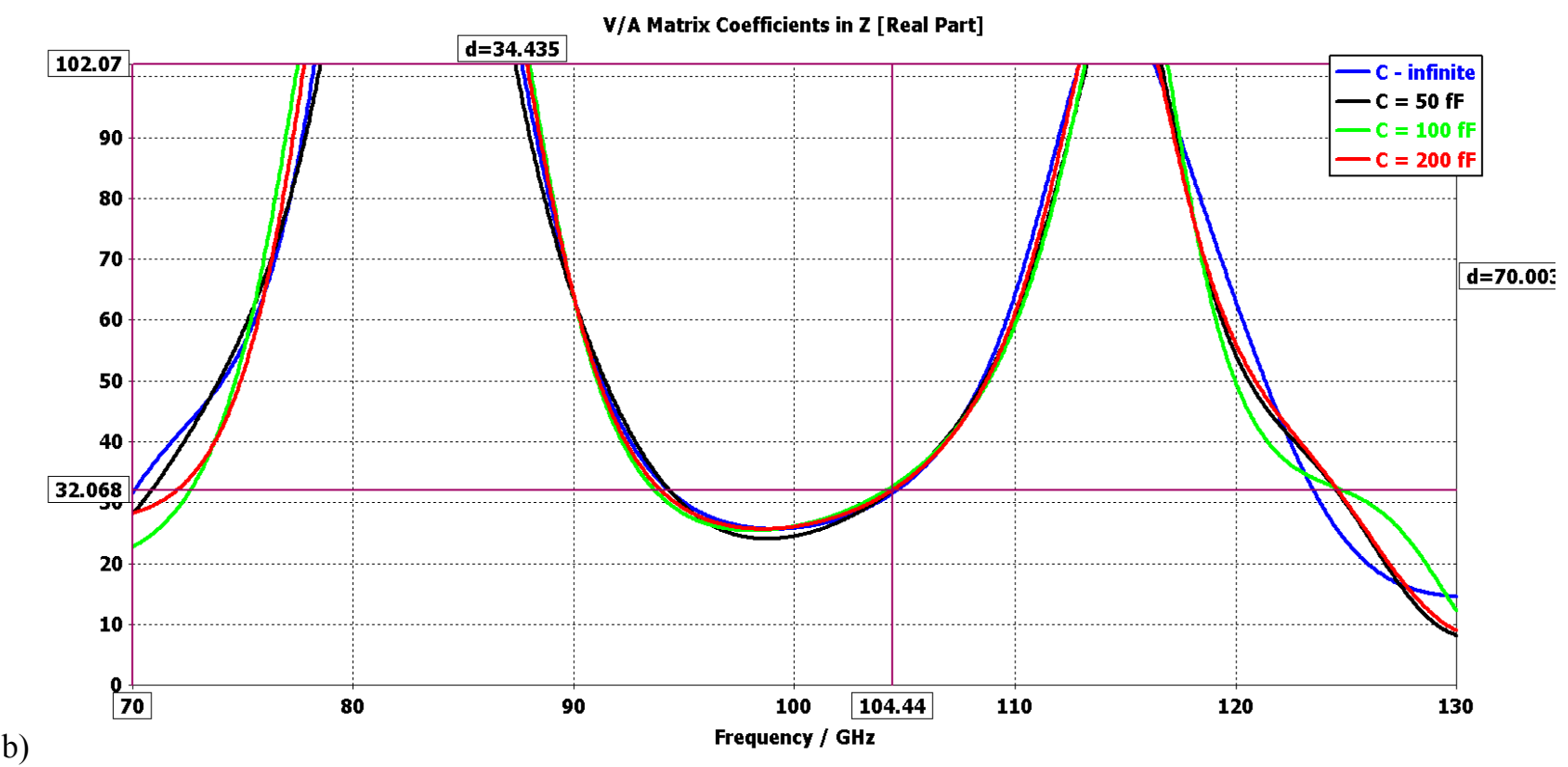

b)

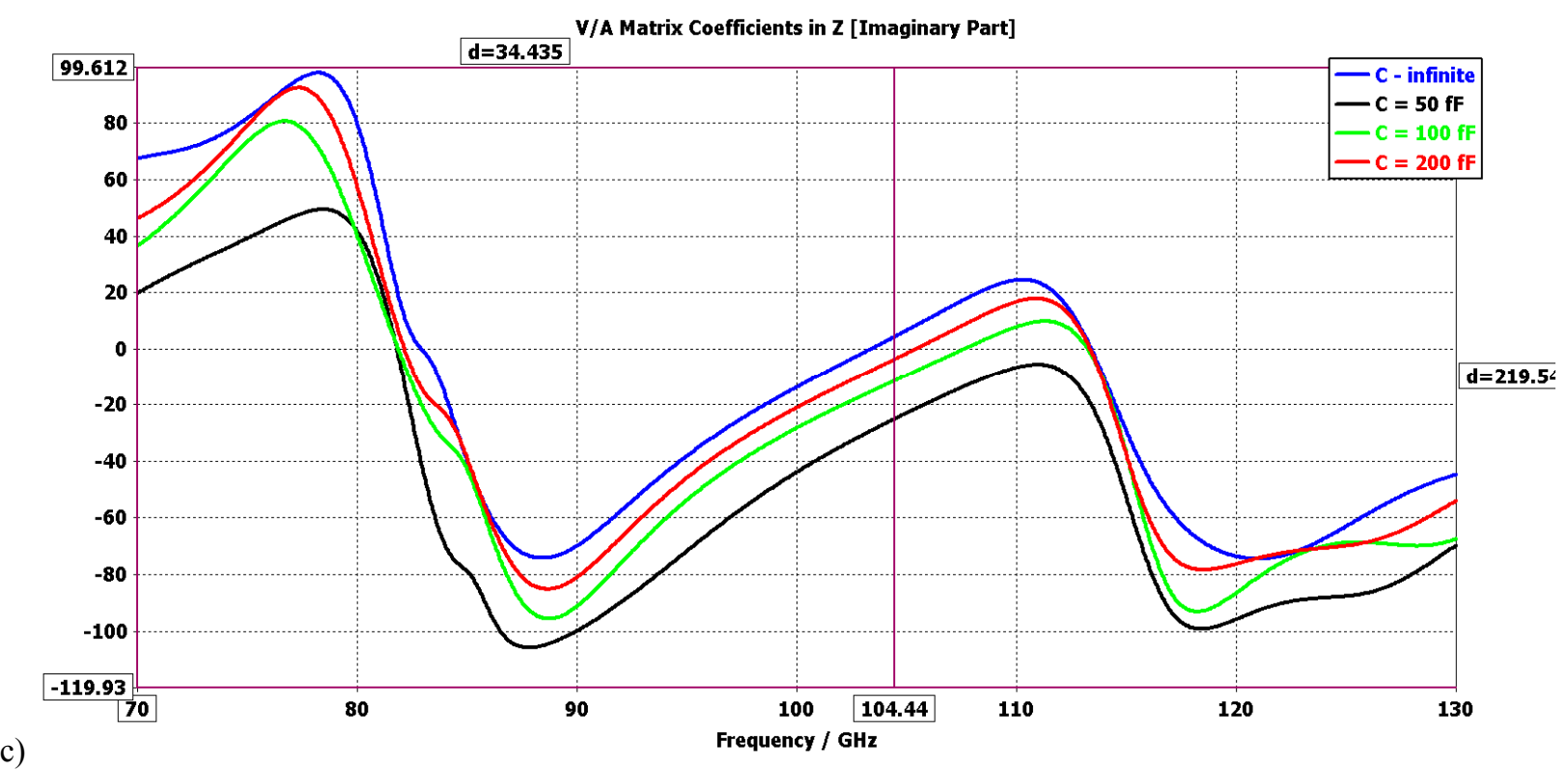

Figure 7. The influence of CEB capacitance on resonance characteristics. (a) S-parameter; (b) ReZ; (c) ImZ, plotted at various $\mathrm{CEB}$ capacitances shown on the right in figure caption in $\mathrm{fF}$.

\section{CONCLUSION}

A novel type of the multichroic bandpass seashell antenna is studied in this paper. The polarized seashell antenna is arranged with individual pair of slots for each frequency that gives unique opportunities for independent adjusting parameters of antennaswith CEBs. Lumped capacitances are proposed for squeezing slots and getting perfect ellipticity due to decreasing the slot length and more freedom in mutual position of slots to realize $\lambda / 2$ distance between slots. The seashell antenna gives an opportunity to select needed bandwidth using resonance properties of slots. For bandwidth of $20 \%$ the optimal configuration proved to be a seashell $\lambda / 2$-slot antenna with lumped capacitances and MSLs with 
CEB. This configuration is using resonant properties of slot antenna itself with MSLs. Matching to microstrip lines and CEBs can be done by tuning position of stubs relatively to the ends of slots. The seashell antenna has possibility of easy expansion to more than two frequencies by insertion of the next loops inside previous ones.

\section{ACKNOWLEDGEMENTS}

This work is supported by the Russian Ministry of Science under Project 3.2054.2014/K. The authors would like to thank the ESA collaboration on multichroic systems for stimulation of this work, P. de Bernardis, A. Ghriby, A. Sobolev, M. Tarasov, A. Pankratov, A. Gordeeva and A. Mukhin for useful discussions.

\section{REFERENCES}

[1] BICEP2 collaboration, Phys. Rev. Lett. 112, 2014101, astro-ph/1403.3985 (2014).

[2] Planck and BICEP2/Keck coll., Physical Review Letters, 114, 10, 101301, astro-ph/ 1502.00612 (2015).

[3] The ESA Tender ESTEC ITT AO/1-7256/“Next Generation Sub-millimetre Wave Focal Plane Array Coupling Concepts", February 2013.

[4] L. S. Kuzmin., A, Chiginev, E, Matrozova, and A. Sobolev. "Multifrequency Seashell Slot Antenna With ColdElectron Bolometers for Cosmology Space Missions," IEEE Transactions on Applied Superconductivity Volume: 26 Issue: 3, pp, 1-6 (2016)

[5] R. O'Brient et al., "A dual-polarized broadband planar antenna and channelizing filter bank for millimeter wavelengths," Appl. Phys. Lett. 102, 063506 (2013);

[6] L. S. Kuzmin. "A Resonant Cold-Electron Bolometer With a Kinetic Inductance Nanofilter", IEEE TRANSACTIONS ON TERAHERTZ SCIENCE AND TECHNOLOGY, VOL. 4, pp 314-320, (2014).

[7] G. Chattopadhyay, D. Miller, H.G. LeDuc, and J.Zmuidzinas, IEEE Trans. on Microwave Theory andTech., 48, $1680(2000)$.

[8] L. Kuzmin, "Optimization of the Hot-Electron Bolometer for Space Astronomy," SNED Proc., pp. 145-154 (2002).

[9] L.S. Kuzmin. "Cold-Electron Bolometer," in book: BOLOMETERS, ed. A.G.U. Perera, intechweb.org, ISBN 978-953-51-0235-9, pp. 77-106 (2012).

[10] M.Tarasov, L.Kuzmin, V.Edelman, P. de Bernardis, Optical Response of a Cold-Electron Bolometer Array Integrated with a 345-GHz Cross-Slot Antenna, IEEE Trans. on Appl. Supercond.,21, 3635 (2011). 